\title{
EL NEGOCIO ÉTNICO, NUEVA FÓRMULA DE COMERCIO EN EL CASCO ANTIGUO DE MADRID. EL CASO DE LAVAPIÉS
}

\author{
POR \\ JUAN A. CEBRIÁN DE MIGUEL

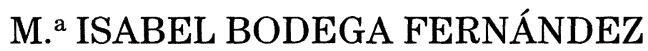

\section{Introducción}

La población extranjera residente en España, compuesta por unos miles de personas en los años 70, supera actualmente el millón de personas: 1.100.000 residentes extranjeros a finales del 2001 (Anuario Estadístico de Extranjería, 2001), que representan el 2,75\% de la población total. Los jubilados de la Europa desarrollada, tan importantes hace 20 años, han tenido que ceder el paso a otros individuos cuyo interés en España no radica en su clima soleado y sí, en cambio, en las posibilidades de trabajo que ofrece.

Este segundo colectivo de inmigrantes desencadena procesos de reagrupación familiar, aparición de segundas generaciones, y aumento de las tasas de naturalización. Como resultado, asistimos a la consolidación de comunidades étnicas en las zonas de mayor densidad de inmigrantes, como es el caso de la ciudad de Madrid, en general, y de alguno de sus barrios, en particular. Embajadores, uno de los seis barrios del distrito Centro de Madrid, es el marco administrativo de Lavapiés: judería, primero, lugar castizo, por antonomasia y enclave étnico - o crisol de razas, como se prefiera - en la más estridente actualidad.

Juan A. Cebrián de Miguel y M. ${ }^{a}$ Isabel Bodega Fernández: Departamento de Geografía. Instituto de Economía y Geografía, CSIC.

Estudios Geográficos, LXIII, 248/249, 2002 


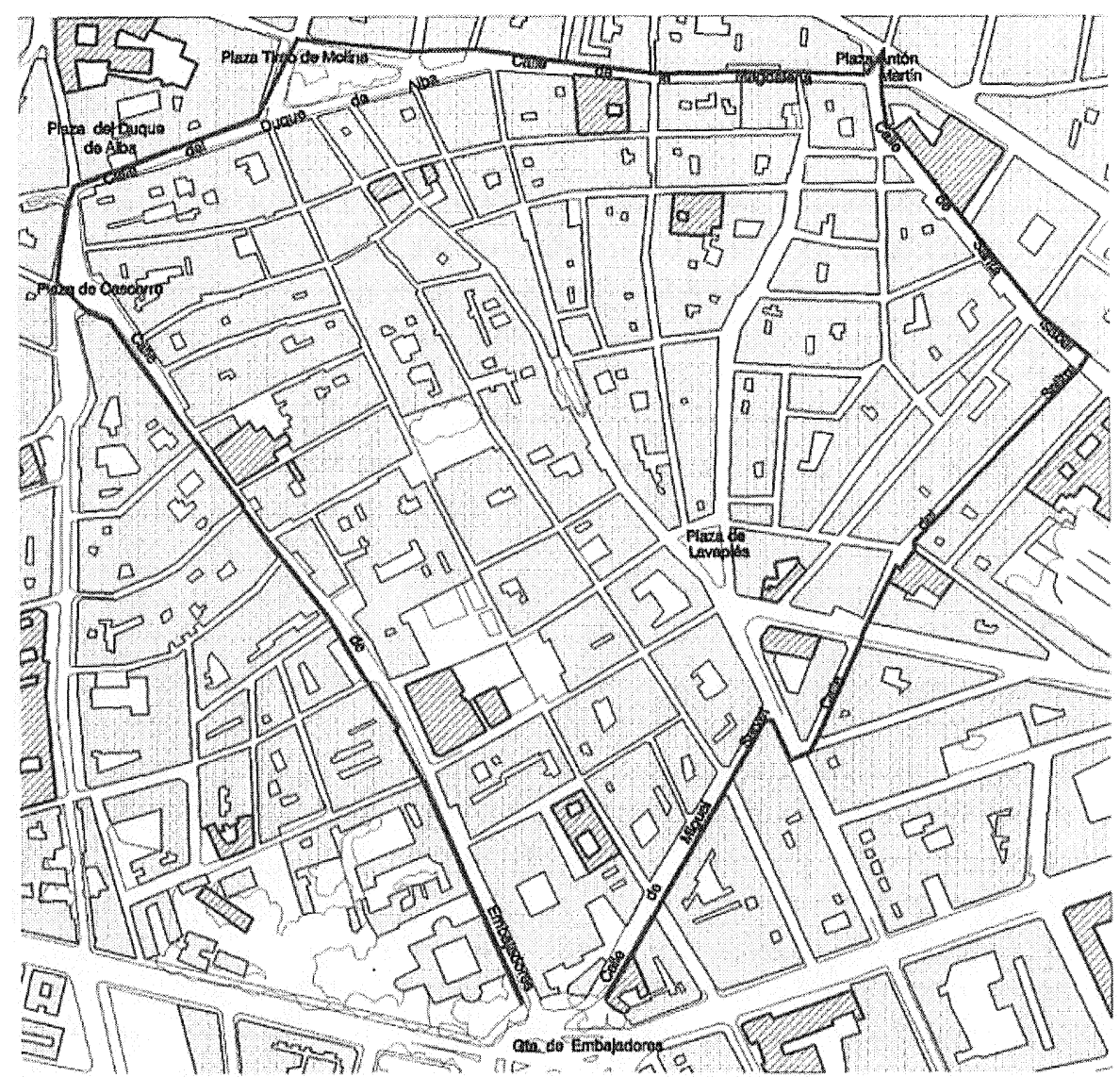

Lavapiés. Delimitación de la zona de estudio.

Llevan el nombre de Lavapiés una calle y una plaza específicas, y un conjunto de manzanas indeterminado. Por este motivo, hemos tenido que delimitar, provisionalmente, nuestra zona de estudio (ver gráfico), recurriendo al trazado de las calles más transitadas y a los aspectos más llamativos de la morfología inmobiliaria. Al contrario de lo que pudiera parecer, la palabra procede muy probablemente del hebreo «aba-puest» (lugar de judíos, judería), contrastando con la etimología del nombre del barrio: Embajadores (del latín medieval «ambactia», misión), por tratarse de la zona que atravesaban los embajadores para presentar sus credenciales ante la Corte del Rey de España.

Estudios Geográficos, LXIII, 248/249, 2002

$$
-560-
$$


Nuestra zona de estudio termina, al norte, en la línea que forman la calle Duque de Alba, el lado sur de la plaza triangular de Tirso de Molina, y la calle de la Magdalena. Al este, en descenso topográfico, el límite es la calle de Santa Isabel. Al oeste, la calle de Embajadores, desde la plaza de Cascorro hasta la glorieta de Embajadores, separa Lavapiés del Rastro. Aunque la frontera sur, que el callejero sugiere, es la Ronda de Valencia, la tipología urbana nos ha llevado a optar por una línea quebrada compuesta por las calles de Miguel Servet, Valencia y del Salitre.

En los últimos años, los comercios regentados por inmigrantes extracomunitarios han pasado a formar parte del paisaje urbano de la zona de Lavapiés, dando paso a una nueva imagen del inmigrante como categoría social de gran heterogeneidad. La mayoría de los comercios étnicos son llevados por familiares o en colaboración con personas de estrecha confianza que, en muchos casos, trabajan sin contrato laboral. Las clases populares consideran que estos negocios de inmigrantes constituyen un elemento revitalizador de los barrios degradados del casco antiguo de la ciudad.

En nuestro trabajo de campo en la zona de Lavapiés, hemos contabilizado, a lo largo del año en curso, 415 establecimientos comerciales regentados por extranjeros, en su mayoría chinos, bangladeses, pakistaníes, iberoamericanos, magrebíes, subsaharianos, etc. La apertura de este tipo de negocios étnicos se ha producido en un contexto de larga crisis del comercio familiar tradicional autóctono, que ha estado, incluso, cerrado durante un tiempo, y ha sido revitalizado gracias a este «nuevo concepto comercial».

Al mismo tiempo, y como consecuencia de este fenómeno, en las zonas donde es mayor la incidencia de comercios étnicos, han proliferado los alquileres de viviendas de bajo nivel económico, donde residen un alto número de inmigrantes y tienen su sede diversas instituciones culturales vecinales, lo que ha dado lugar a verdaderos enclaves étnicos. Nos enfrentamos con un proceso que se percibe en la fisonomía particular de viviendas, locales comerciales y vías públicas, porque provoca significativas alteraciones en el espacio, en la identidad y en la economía de los barrios afectados.

Entre los cambios más importantes de finales de siglo xx en el mercado de trabajo en Europa y Norteamérica se encuentra el alto grado de trabajadores pertenecientes a las minorías étnicas que se definen como autoempleados. Si bien es cierto que las sociedades occidentales han ex-

Estudios Geográficos, LXIII, 248/249, 2002 
perimentado un tránsito en el empleo, desde el trabajo en las grandes corporaciones a la inserción laboral en empresas de medianas o pequeñas dimensiones, este proceso ha sido más marcado entre los ciudadanos y residentes de origen extranjero (véase Barret, G; Jones, T y McEvoy: 1996). Este hecho ha despertado la curiosidad de los expertos, marcando así una de las vías de incorporación al estudio de las economías étnicas.

La incorporación de la variable «étnica», en los estudios sobre autoempleo y economía urbana, puede ser explicada como resultado de la combinación de dos factores sobresalientes. No debemos olvidar que, desde los años setenta, la presencia de minorías étnicas visibles en los países clásicos de inmigración se dispara por la desintegración de las políticas racialmente selectivas. En Europa es, quizá, el cierre de fronteras y la estabilización de los flujos, lo que determina la nueva composición de las comunidades de origen inmigrante. Los estudios en economía étnica se enmarcan, por tanto, en dos condiciones sociales existentes. En primer lugar, la dinámica migratoria de la mayor parte de los países occidentales, una dinámica cuyos rasgos más señalados han sido el asentamiento y la conformación de comunidades de origen extranjero étnicamente visibles. La economía étnica que florece en muchas ciudades europeas y norteamericanas es el resultado de una instalación con vocación de permanencia que afecta, entre otras, a las estrategias de inserción laboral. El segundo elemento hunde sus raíces en los procesos de reconversión de las economías urbanas en la mayor parte de las sociedades postindustriales, donde la economía étnica se vislumbra como un factor de cambio de los contornos geográficos y sociales de ciertos barrios y distritos, así como por su impulso en la configuración de los llamados enclaves étnicos.

En el caso español, las iniciativas empresariales de esta naturaleza corren parejas a los procesos de instalación definitiva de los inmigrantes y es resultado del paulatino proceso de maduración del fenómeno, si bien, la creación de restaurantes entre la comunidad china y de pequeños negocios de alimentación y de electrónica entre los hindúes, en el caso específico de las Islas Canarias, tiene cierta tradición. Las iniciativas empresariales étnicas se multiplican en nuestro país desde mediados de los años noventa, encontrándose, en la actualidad, en plena expansión. Es evidente, por ejemplo, el proceso de transformación económica y geográfica de ciertos barrios como el de Lavapiés en Madrid o el Raval de Barcelona.

Estudios Geográficos, LXIII, 248/249, 2002

$$
-562-
$$




\section{Negocio étnico}

Los estudios sobre Economía étnica se desarrollan a partir de los años setenta con las aportaciones pioneras de Light. Este autor norteamericano destaca uno de los puntos de partida de las investigaciones realizadas con posterioridad: la alta propensión al autoempleo entre los individuos de origen extranjero (en su caso en Estados Unidos). Esta pauta «laboral» puede ser calificada como una «anomalía», si se pone en comparación con lo que observamos en el caso de las poblaciones de la sociedad de acogida.

Los estudios de Bonacich ahondan en esta cuestión y sostienen que las iniciativas empresariales étnicas deben ser vistas, en primera instancia, como una adaptación reactiva ante los procesos de remoción geográfica y cultural (véase Bonacich, E.: 1973). La creación de negocios es entendida, desde este planteamiento, como una reacción ante el bloqueo de las oportunidades en el mercado de trabajo, actuando el autoempleo como una especie de estrategia de supervivencia vinculada a los lazos de solidaridad existentes dentro de la comunidad. En conclusión, una gran parte de las investigaciones realizadas durante los años setenta pretenden dilucidar las causas por las que, aún entre algunas minorías que durante generaciones experimentaron procesos de discriminación se aprecia un elevado porcentaje de individuos que tienen negocios en propiedad. La creación de negocios y comercios entre los inmigrantes fue, en un primer momento, explicado, en exclusiva, como resultado de la discriminación que sufrían los recién llegados en los mercados de trabajo locales viéndose forzados a buscar nuevos nichos laborales. Este planteamiento de partida sufre múltiples cambios posteriormente.

En contra de las interpretaciones más habituales que vinculan el florecimiento de la economía étnica con la estabilización de las comunidades, Bonacich considera que la propensión a la inversión de ciertas comunidades está motivada por la sensación de transitoriedad en el proyecto migratorio. El mito del retorno impulsa, en su opinión, un tipo de actitud instrumental en el que el país de destino donde esté no es visto como un nuevo hogar o como un lugar donde establecer nuevas relaciones sociales, sino como un medio para hacer dinero rápidamente y retornar cuanto antes al país de origen (veáse Barret, G.; Jones, T. y McEvoy: 1996). Los estudios pioneros también resaltan que las especiales dificultades de inserción laboral de los inmigrantes propician el desarrollo 
de actividades económicas en nichos marginales rechazados por las poblaciones autóctonas. Las condiciones de trabajo de este tipo de negocios se caracterizan por su especial dureza, sobre todo en el aspecto de la duración de la jornada laboral: la base de la competitividad de muchos de estos negocios. De ahí la importancia de la solidaridad y del trabajo familiar en estas iniciativas. El éxito de este tipo de negocios se encuentra, además, en muchos casos, en la fidelidad de los clientes, muchos de ellos, pertenecientes a la misma comunidad de origen.

La investigación en esta materia durante los años ochenta hace mayor hincapié en los valores empresariales, el capital humano y el apoyo familiar y comunitario como elementos determinantes del establecimiento de negocios étnicos. De ahí un enfoque que subraya los «valores comunitarios y culturales» como fuente de la explicación de este tipo de pauta. $\mathrm{Y}$ es que uno de los resultados más sobresalientes de los estudios sobre economía étnica es la diferencia existente en el nivel de autoempleo y de creación de negocios entre comunidades procedentes de diversas cuencas migratorias. En el caso anglosajón es de destacar la pauta contrapuesta entre las comunidades de origen asiático y las procedentes del Caribe. Los «recursos étnicos y culturales» aparecen como los elementos determinantes de la generación de empleo en el caso de las comunidades de origen extranjero, que alcanza, en ciertos países, una tasa que supera a la observada entre la población autóctona. De hecho, la gran idea de las investigaciones en economía étnica, en el sentido de aquella que ha gozado de mayor éxito entre los expertos es que, como afirma Jones y sus colaboradores, el factor cultural anula las condiciones materiales y propicia un comportamiento económico que se desvía de la norma: los atributos culturales de ciertos grupos minoritarios explican y determinan la tendencia a la inversión y a la creación de negocios propios. Las comunidades asiáticas son presentadas, a menudo, como ejemplos paradigmáticos.

Como ha señalado Monder y sus colaboradores, este tipo de interpretaciones «culturalistas» han sido cuestionadas por investigaciones más recientes, en las que se hace mayor hincapié en la estructura de oportunidades a las que tienen que hacer frente este tipo de colectivos. Durante la década de los noventa, los estudios han adoptado, en el mundo anglosajón, un enfoque más «interaccionista», en donde se destaca la interconexión entre los recursos internos de los que disfruta la comunidad y la estructura externa de oportunidades.

Estudios Geográficos, LXIII, 248/249, 2002

$$
-564-
$$


En esencia, lo que se considera un modelo cultural de interpretación de la economía étnica subestima el impacto que la discriminación racial puede tener en el desarrollo de estrategias grupales y en la dependencia de recursos «comunitarios» para sobrevivir (Monder, R., et al.: 2000). Son similares las conclusiones de uno de los mayores expertos europeos en esta materia. Jan Rath, en su inspección del caso holandés, subraya que los análisis han reducido el fenómeno del negocio étnico a un fenómeno de naturaleza etnocultural que subestima el marco económico e institucional. Las investigaciones recientes, sin embargo, ponen más atención en los factores de transformación estructural que están teniendo lugar en las economías urbanas, así como en el marco institucional del Estado del Bienestar en el que opera la inversión y la formación de negocios y en el impacto del mercado internacional en este tipo de negocios ${ }^{1}$ ( Rath, J.: 2000).

Aunque las posiciones de tipo culturalista han perdido preeminencia en los estudios, es cierto también que la variable cultural sigue siendo incorporada, debido, quizá, al especial carácter de este tipo de negocio. Los estudios han desarrollado, por ejemplo, líneas de argumentación en torno a dos ejes fundamentales: a) en primer lugar, cuáles serían los elementos que diferencian a las distintas comunidades étnicas en el proceso de creación de este tipo de establecimientos y b), en segundo lugar, cuáles son los elementos diferenciadores de la actividad empresarial de las minorías respecto a la de los nacionales en el mismo tipo de negocios. Como indica Light para el caso estadounidense, aparecen una docena de comunidades étnicas en las que el nivel de autoempleo supera significativamente la media nacional. Además de los judíos (el ejemplo quizá más estereotipado), los individuos procedentes de China, Japón y Corea presentan los porcentajes más elevados, al igual que hindúes y paquistaníes en el caso británico (Light, I.: 1984). Las investigaciones posteriores han destacado, sin embargo, que las razones que motivan la «inversión étnica» son muy variadas y no se ven originadas, en exclusiva, por los procesos de discriminación o de prácticas culturales (véase Portes, A: 1995).

La creación de negocios en ciertas comunidades étnicas ha propiciado procesos de movilidad social ascendente entre determinados seg-

${ }^{1}$ Véase Sassen, S. (1991) y Kloosterman, R. (1996).

Estudios Geográficos, LXIII, 248/249, 2002 
mentos de esas comunidades. De hecho, en Estados Unidos, los ingresos medios de los empresarios étnicos, así como de los autónomos, superan a los trabajadores por cuenta ajena (Portes, A: 1995). El resultado ha sido, en buena medida, la conformación de una nueva clase, de pequeños propietarios de negocios, en el caso de estas comunidades sociales, que se encuentran en un punto intermedio de la escala social ${ }^{2}$ y que, a través de los ingresos que les proporcionan sus negocios, han propiciado:

a) Una mejora de las condiciones de vida de sus descendientes: acceso a estudios universitarios e incremento de la cualificación de las segundas generaciones.

b) Procesos de creación de riqueza y de creación de empleo en el ámbito urbano.

c) Creación de enclaves étnicos y de comunidades influyentes en la vida social y política del país de acogida: conformación de grupos de presión a través de la riqueza material y el poder social que proporciona la inversión.

\section{Delimitación de objetivos y conceptos}

En su sentido más amplio, la economía étnica podría definirse como el conjunto de actividades productivas de los residentes extranjeros pertenecientes a minorías significadas. En casos como el español, la aportación económica fundamental de los inmigrantes consiste en su participación como trabajadores por cuenta ajena. En los países occidentales con una tradición inmigratoria mayor (EEUU, Canadá, Alemania, Reino Unido, Países Bajos, Bélgica, Francia o Australia) la iniciativa étnica empresarial, cuyo primer escalón es el trabajo por cuenta propia, se hace progresivamente más notoria. En el caso español, el autoempleo y la instalación productiva de los inmigrantes en forma de empresa étnica sólo han crecido significativamente a partir de mediados de los noventa, como hemos afirmado previamente.

Por definición, la propiedad de una empresa étnica estará, total o parcialmente, en manos de inmigrantes extranjeros. Esta clase de negocio puede estar orientado tanto a mercados de minorías, como al público en

${ }^{2}$ Véase el concepto de «middleman minorities» de Bonacich. 
general y tener una proyección local, regional, nacional o internacional. Es el caso del impacto de las comunidades transnacionales en el establecimiento de algunos negocios en los nuevos países de inmigración del sur de Europa. Así mismo, este tipo de empresas pueden ubicarse en muy distintos sectores económicos y reunir a un número muy diverso de empleados. En España, debido a que se trata de un fenómeno reciente, encontramos empresas étnicas en zonas urbanas, con una proyección local o regional en el sector servicios y con un número reducido de empleados.

\section{Trabajo autónomo y economía sumergida}

Es importante subrayar el concepto de trabajador autónomo (contabilizado en los registros oficiales de la mayor parte de los países receptores como parte de los trabajadores que desempeñan su actividad laboral en empresas étnicas) por la relevancia que tiene para nuestro trabajo de investigación. Por tal, puede entenderse el sujeto económico que, en su trabajo, generalmente individual, aporta por sí mismo los factores de producción, es decir, mano de obra y capital. Esta afirmación, sin paliativos, debe tomar en consideración la heterogeneidad interna de situaciones que se producen, de hecho, entre los trabajadores autónomos: por ejemplo, la subcontratación y dependencia respecto a unidades empresariales de carácter multinacional. La proliferación de los trabajadores autónomos, uno de los fenómenos del autoempleo, puede estar ligada a la economía sumergida. Este último concepto se define como aquella parte de la actividad económica, que varía entre el 10 y el $30 \%$ sobre el PIB oficial, que no entra en los circuitos estadísticos y fiscales. Sus causas fundamentales son eludir la presión fiscal e incumplir las cargas de la seguridad social y determinadas reglamentaciones laborales. La economía sumergida coincide con situaciones de gran volumen de paro.

El fenómeno del autoempleo está muy relacionado con el mercado de trabajo no declarado. Se trata de un tema importante para todos los Estados miembros de la Unión Europea, y por esta razón, la Comisión Europea, a finales de 1993, abogaba por ampliar el concepto de trabajo y urgía a los Estados a facilitar «la reincorporación al mercado de trabajo de muchos trabajadores actualmente marginados o que realizan trabajos clandestinos». 
La definición de trabajo no declarado es el de «cualquier actividad retribuida que sea legal en cuanto a su naturaleza, pero que no sea declarada a las autoridades públicas». Está claro que se excluyen las actividades delictivas y el trabajo no cubierto por el marco normativo habitual cuya declaración a las autoridades no sea obligatoria.

El motivo para que los empresarios, empleados y trabajadores, inmigrantes o nativos, trabajen en la economía sumergida no es otro que el económico. Hay posibilidad de aumentar las ganancias, evadiendo los impuestos sobre los ingresos y evitando las cotizaciones sociales, con lo que se reducen los costes de producción.

Resulta imposible determinar el número de ciudadanos de terceros países que residen en la U.E. de forma clandestina y que participan en la economía sumergida ${ }^{3}$. Uno de los efectos del trabajo no declarado para los inmigrantes es la ausencia de derechos a los seguros de salud, de pensión de jubilación, y de accidentes de trabajo y enfermedades profesionales o comunes. Los inmigrantes en esta situación no están cubiertos por el sistema de protección social del país de acogida, viéndose excluidos de los beneficios de un contrato formal (laboral o mercantil), tales como: la formación profesional, un perfil específico de carrera, los incrementos salariales, el sentimiento de pertenecer a una empresa, etc. Es muy importante fomentar ayudas para el trabajo realizado por los inmigrantes en el sentido de que puedan trabajar como autónomos, o incluso crear empleo para sus compatriotas o para los autóctonos.

\section{Redes migratorias familiares: su dimensión económica}

Son muchas las interpretaciones del fenómeno migratorio desde la Economía. Así, el enfoque microeconómico sostiene que el objetivo del inmigrante es el de maximizar su bienestar, para lo cual comparará su situación presente con la futura y, dependiendo del balance entre los beneficios y los costes, tomará la decisión de salir, o no, de su país. Este modelo clásico de inmigración considera que la decisión de migrar se produce como consecuencia exclusiva de las diferencias salariales entre los países de destino y de acogida.

${ }^{3}$ En España el análisis de la inmigración irregular se ha basado fundamentalmente en el estudio de los registros que arrojan las diversas regularizaciones y el contingente anual.

Estudios Geográficos, LXIII, 248/249, 2002

$$
-568-
$$


Sin embargo, deben ser tenidos en cuenta otros aspectos. Cuanto más tiempo tarde el inmigrante, o su familia, en percibir la mejora en su bienestar derivada de su decisión de migrar, más importancia tendrá el coste presente ocasionado por el hecho de emigrar. Otras interpretaciones, como la teoría de la Nueva Economía de la Inmigración y la Teoría de Redes Sociales, hacen hincapié en los vínculos y las relaciones familiares a la hora de la adopción de la decisión migratoria, la instalación en el país de destino y las estrategias laborales y profesiones adoptadas en la sociedad de acogida. En consecuencia, parece que puede establecerse una relación intensa entre la decisión de migrar y la estrategia familiar, atribuyendo a la familia la toma de decisiones, como parte de una estrategia diseñada a complementar los ingresos familiares y evitar el deterioro de su nivel de vida. Mediante la emigración de uno de sus miembros, la familia diversifica sus riesgos, ya que invierte, a la vez, en un número de mercados distintos, compartiendo los costes de financiación del viaje y los beneficios derivados del envío de remesas. Por lo tanto, el fenómeno de la emigración puede ser explicado a partir de la optimización de la aversión al riesgo. Este planteamiento considerado, supone explicar la emigración, no como una situación de desesperación o deseo de aventura, sino como una estrategia de la familia encaminada a reducir el riesgo de pauperización en el país de origen, tratando de diversificar sus fuentes de ingresos a través de situar a uno o varios de sus miembros más capacitados en un mercado distinto e independiente, así como el inicio de ciertas iniciativas empresariales que utilicen, en parte, recursos humanos y materiales procedentes de la familia extensa y de la comunidad de origen.

Diversos estudios de campo han subrayado que, en un primer momento, la familia se convierte en la fuente de financiación del traslado, generando los recursos necesarios para soportar tanto los gastos del desplazamiento como los costes iniciales derivados de la instalación en un período de alto riesgo para el inmigrante. De esta forma, contribuyen a reducir esos riesgos. Esta inversión debe ser compensada en el medio-largo plazo a través de las remesas, que garantizan una cierta estabilidad financiera para la familia del emigrante, contribuyendo a aumentar su bienestar. Estaríamos, en consecuencia, ante un pacto o acuerdo entre el inmigrante y su familia que genera obligaciones y beneficios para ambas partes y que consolida y refuerza las comunidades trasnacionales. Este planteamiento explicaría perfectamente el comportamiento de la comu- 
nidad china en España, a partir de las facilidades crediticias que las propias familias del inmigrante le proporcionan, y el compromiso de devolución de dichos capitales, así como de la colocación en el negocio étnico de otros familiares ${ }^{4}$.

Los inmigrantes económicos proceden generalmente de sociedades tradicionales o en transición, teniendo un valor fundamental los comportamientos familiares. En un primer nivel, el cabeza de familia, que comparte su renta con los miembros de su familia, lo que hace es una redistribución de sus recursos económicos entre sus beneficiarios, por lo que se comporta como un «inmigrante altruista». En un segundo nivel, la familia inmigrante y los colectivos de un mismo país facilitan la búsqueda y el encuentro efectivo de trabajo para los recién llegados, o bien para las segundas generaciones en edad laboral. En un tercer nivel, hay que señalar que, en determinados colectivos, la familia actúa creando autoempleo de capital étnico, concediendo ayudas a los familiares, y aportando los asalariados de las empresas familiares. Este sería, por ejemplo, el caso del colectivo chino en nuestro país. De esta forma, la unión de la familia inmigrante palia en muchas ocasiones el problema del desempleo y la precariedad en el empleo o subempleo (tanto en los países de origen, por el reenvío de remesas, como en los de acogida) o, dicho de otro modo, el sentimiento altruista del trabajador inmigrante ayuda a sus beneficiarios, entendiendo por tales a los miembros de su familia. Por ejemplo, en el colectivo chino se dan circunstancias en las que los individuos jóvenes en edad de trabajar ofrecen actividades lúdicas y de refuerzo a los hijos menores de inmigrantes. Los objetivos de estas actividades son favorecer la autoestima y la solidaridad, para evitar el desarraigo, asumiendo la diferencia cultural y racial como un factor enriquecedor. Con ello se trata de resaltar la importancia de compensar las carencias educativas detectadas en los menores.

Este sentimiento de unidad, de altruismo y de ayuda mutua nos lleva a plantearnos el concepto de capital social y a considerarlo en nuestro análisis, un concepto que alude a la capacidad de las personas de agruparse y organizarse para lograr propósitos comunes. Esa capacidad de

\footnotetext{
${ }^{4}$ Las sociedades tradicionales y en transición se caracterizan porque la familia palia el peso de los jóvenes, ancianos y personas enfermas, actuando fundamentalmente en tres niveles:a) como unidad de convivencia y de consumo, b) como unidad de información y gestión de empleos y c) como unidad productiva de bienes y servicios.
}

Estudios Geográficos, LXIII, 248/249, 2002 
asociarse depende del grado en que las comunidades humanas comparten normas y valores que hacen que los intereses individuales y personales pasen a un segundo plano. Es en la familia donde se desarrollan relaciones de gran lealtad y de altruismo entre sus miembros, especialmente en las familias tradicionales extensas, compuestas por abuelos, padres, tíos, primos, hijos, etc., que se ayudan mutuamente.

La familia es, también, una unidad productora de bienestar social, como resultado final de la conjunción de factores materiales, económicos y sociales, que sitúan a los individuos en posiciones de comodidad, valorada en términos comparativos (sincrónicos y diacrónicos).

El altruismo domina el comportamiento en la familia, quizá con la misma profundidad con que el egoísmo predomina en las transacciones de mercado, si bien aquél es mucho más importante en la vida económica de lo que comúnmente se ha venido considerando, ya que es causa, en muchas ocasiones, de que una persona pueda emigrar a otro país. Esta decisión debe ser considerada como un acto de responsabilidad hacia su familia, por cuanto en muchas ocasiones se produce un sentimiento de desesperación por no poder mantener a sus hijos, aún cuando la inmigración suponga, al mismo tiempo, importantes costes psíquicos, de nostalgia y desarraigo.

El aumento de la renta del inmigrante-altruista en ocasiones no produce un aumento del gasto en su propia familia, sino del ahorro, con el objetivo de invertir en formación de capital, lo que en muchas ocasiones determina la apertura de su propio negocio, que denominamos de capital étnico. Como ejemplos más significativos de comercios étnicos en nuestra sociedad, debemos citar las carnicerías de carne de cordero de marroquíes, los restaurantes chinos, las tiendas de alimentación, etc., tal y como veremos a lo largo de nuestro estudio.

\section{Propuesta de investigación}

El objetivo general de esta investigación ha sido analizar la economía étnica que ha aparecido en España a lo largo de la década de los 90. Nuestro análisis incluye dos fases principales: En la primera, se delimitan las dimensiones del fenómeno en términos cuantitativos. En una fase posterior, se pretende construir una tipología de las empresas étnicas en

Estudios Geográficos, LXIII, 248/249, 2002

$$
-571-
$$


nuestro país, a partir de distintas variables (sector económico, dimensiones y tipo de negocio, comunidad a la que se vinculan, etc.) a partir del análisis de los datos secundarios y las conclusiones del trabajo de campo. Esta tipología permite clasificar enclaves y determinar especificaciones productivas de ciertas comunidades que pueden ser utilizadas en investigaciones posteriores. En este proyecto, el análisis de la empresa étnica tiene siempre en cuenta la existencia de redes sociales, y las estrategias integradoras y productivas de las que estas empresas son resultado.

Como el propósito de analizar en profundidad la economía étnica en todo nuestro país supera las posibilidades de esta investigación, nuestro análisis se ha centrado en el estudio de un enclave étnico en el interior de la ciudad de Madrid: el caso de Lavapiés. Además, este estudio puede servir para contrastar algunas hipótesis clásicas:

La creación de empresas étnicas está impulsada por dos factores: la discriminación racial en el mercado de trabajo a la que están sometidos los inmigrantes (efecto disuasor de integración en el mercado laboral de la sociedad de acogida), y la existencia de redes familiares y de solidaridad étnica que facilitan la inversión y aseguran la distribución de servicios y productos. A través de estas empresas, los inmigrantes no sólo se emplean a sí mismos, sino también a miembros de su comunidad y, en ciertos casos, a población autóctona.

La empresa étnica puede ser considerada un factor de integración social, desde la perspectiva de que el autoempleo sea una iniciativa que propicie la inserción laboral (uno de los primeros pasos de la integración social en sus múltiples dimensiones), de transformación productiva (por su impacto en la creación de nuevos sectores o en la caracterización de ciertas economías urbanas), y de cambio en la fisonomía de diversos espacios. En ciertos casos, la economía étnica puede llegar a recuperar áreas urbanas deterioradas, convirtiéndolas en centros de atracción de población o de turistas, como se ha observado en el caso de ciertos barrios de algunas ciudades europeas (Bruselas, Londres, etcétera).

En relación con la propia comunidad, la economía étnica supone para los inmigrantes salir del anonimato, al mismo tiempo que les permite mantener su idiosincrasia, acercando las generaciones más jóvenes a la cultura de sus progenitores, jugando un papel principal en el proceso de reproducción cultural.

Estudios Geográficos, LXIII, 248/249, 2002

$$
-572-
$$




\section{Investigación sobre economía étnica en España}

Hasta ahora, la investigación del fenómeno inmigratorio español se ha dedicado a analizar las características socioeconómicas de las comunidades más importantes, las iniciativas políticas dirigidas a la integración social, la erradicación de reacciones xenófobas entre la población o el análisis de la opinión pública. Son pocos los trabajos que estudian las iniciativas económicas desarrolladas por los inmigrantes, de tal manera que no se conocen investigaciones que aborden el tema de las empresas étnicas en España. Existen, sin embargo, algunos estudios que sí han analizado parcialmente algunas experiencias. Este es el caso de algunos trabajos sobre la comunidad china, que tratan indirectamente el fenómeno de la creación de negocios como estrategia vinculada a su instalación (Beltrán, 2000), o de otros que lo abordan en los análisis sobre el impacto de la inmigración en ciertos barrios de las grandes ciudades (Monnet, 2000). Un estudio reciente sobre el barrio de Lavapiés es, hasta la fecha, una de las pocas investigaciones que afrontan el tema de la presencia económica de los inmigrantes y de los enclaves étnicos (Giménez, 2000).

El análisis de los procesos de inversión productiva de ciertas comunidades étnicas resulta adecuado para profundizar en las estrategias de instalación de los inmigrantes, así como para entender la influencia en ellas de determinados factores, como las redes de solidaridad familiar o social.

Por otra parte, como se pone de manifiesto en algunos cascos históricos, o en determinados barrios de ciertas ciudades españolas, la creación de empresas étnicas en España es un fenómeno en plena expansión, que está transformando productiva y fisonómicamente numerosos espacios.

Nuestro trabajo sirve, además, para combatir ciertos prejuicios que distorsionan la realidad migratoria, como la idea de que la inmigración puede suponer una carga insoportable para los servicios sociales y poner en peligro el modelo de Estado de Bienestar. El análisis de las empresas étnicas en España es una buena muestra de que la inmigración no sólo produce gastos al Estado, sino que, además, es una fuente de riqueza económica.

Las empresas étnicas tienen otra dimensión de gran interés. Su existencia propicia la interacción, la convivencia y la interdependencia entre 
las formas de vida y las identificaciones culturales de la población autóctona de la extranjera. Son, por tanto, un buen instrumento para el desarrollo de sentimientos de tolerancia y de mutuo entendimiento, tan necesarios para alcanzar un espíritu de respeto por el pluralismo y la diversidad.

\section{Técnicas de investigación}

El enfoque de esta investigación hace necesario integrar los métodos y técnicas de investigación, con los aportes teóricos.

Una de las decisiones más importantes del diseño metodológico es la de determinar qué tipo de estrategias y de técnicas resultan más idóneas para nuestro objeto de estudio. Resulta conveniente realizar un análisis de datos secundarios. Se ha decidido, además, utilizar técnicas de investigación cualitativa (entrevistas en profundidad).Empleamos también, para ilustrar nuestra exposición, algunos datos primarios producidos por encuestas españolas en materia de inmigración. En suma, ponemos en práctica la llamada triangulación, o «aproximación multimétodo», como estrategia de investigación.

\section{Datos secundarios: el diagnóstico cuantitativo del fenómeno}

Comenzamos tomando en consideración el nivel de autoempleo, de establecimiento por cuenta propia de los extranjeros en España. Esta cifra puede ser un dígito orientador de la propensión de distintos colectivos a introducirse en el mercado de trabajo español siguiendo estrategias diferentes a las del trabajo por cuenta ajena (Barret, Jones y McEvoy, 1996). Para ello se emplean los datos recopilados cada año por el Ministerio de Trabajo y publicados en el Anuario de Estadísticas Laborales. La disponibilidad de esta fuente permite la elaboración de series temporales, completándose dichos datos con otras cifras: permisos de trabajo concedidos y trabajadores con permisos de trabajo en vigor. Los datos secundarios sirven para el diseño de una serie de tablas y de indicadores relativos, que ponen de manifiesto las diferentes actitudes de los inmigrantes en el plano laboral. Este diagnóstico se completa, en la medida de lo posible, con la utilización de otros datos, como las altas en la

Estudios Geográficos, LXIII, 248/249, 2002

$$
-574-
$$


Seguridad Social recogidas por la Tesorería General de la Seguridad Social (Gerencia de Informática) y disponibles en el OPI (Observatorio Permanente de la Inmigración). De esta fuente se tratan, fundamentalmente, las cifras referidas a trabajadores por cuenta propia y a los autónomos (28,3\% del total).

El análisis de datos secundarios, que estamos realizando en este momento incluye la recogida de información sobre negocios étnicos existentes. Las fuentes fundamentales son los registros del impuesto de actividades económicas de la Cámara de Comercio e Industria y la Comunidad de Madrid. Estos datos se tratan con un programa estadístico con el fin de obtener conclusiones que sirvan para posteriores estudios de carácter comparativo, y para la caracterización de los negocios en el momento de la recogida de datos. Esta caracterización determina, asimismo, el diseño de la muestra estructural, que permite recoger el amplio abanico de negocios existentes en el barrio. También se pretende recopilar otras cifras disponibles en sindicatos, ONGs y organizaciones empresariales, aunque la explotación y el análisis de éstas deberá realizarse con cautela debido a las dificultades: fragmentación, parcialidad y dispersión que suelen presentar este tipo de fuentes.

Además se están recogiendo datos de opinión pública producidos en España por el Centro de Investigaciones Sociológicas (CIS) y el CIRES (ASEP), así como datos europeos publicados en los Eurobarómetros. La finalidad de su consideración en la investigación es contrastar opiniones y realidades (resultados) sobre si la inmigración es, o no, un factor de creación de riqueza.

\section{La investigación cualitativa}

El análisis de los datos secundarios (diagnóstico) debe completarse con la información obtenida mediante la aplicación de técnicas de investigación cualitativa. Las posibilidades interpretativas de este tipo de técnicas permite profundizar en el análisis de la economía étnica local. La realización de entrevistas en profundidad cumple varias funciones. En primer lugar, clarifica los factores de motivación para la inversión, explorando las diferencias por colectivos. De esta manera, se profundiza en las circunstancias que han rodeado el proyecto migratorio, la instalación y la inversión como estrategia de establecimiento: supervivencia económica 
y producción de riqueza. Este análisis nos permite, además, establecer diferentes pautas y tipologías de inmigrantes inversores. En segundo lugar, las entrevistas permiten analizar los negocios étnicos (tipo de mercado al que se dirige, evolución a lo largo del tiempo, prácticas de gestión, capacidad de creación de empleo, etc.).

Las entrevistas en profundidad se han dirigido, en primer lugar, a diversos inmigrantes (empresarios y trabajadores autónomos) para establecer las diferencias entre estos dos tipos de categorías. En una segunda fase las entrevistas se han dirigido a propietarios de negocios étnicos, para profundizar en su experiencia/estrategia de inversión.

La elaboración de una tipología de negocios étnicos no sólo nos permite ofrecer un estado de la cuestión, sino además elaborar proyecciones de estas actividades, los efectos sobre la comunidad, o el impacto sobre determinadas áreas urbanas.

Los negocios étnicos han adoptado diversas formas. Los especialistas en esta materia han realizado diversas tipologías. Quizá la más habitual es la del miembro de una determinada comunidad que proporciona servicios y bienes al resto de los miembros de su comunidad étnica. Así, una buena parte de los productos que venden son importados directamente de los países de origen para cubrir ciertas necesidades específicas que no pueden ser satisfechas por el mercado habitual (ingredientes con los que preparar platos tradicionales, prensa del país de origen, ciertos utensilios domésticos, etc). Este tipo de negocios se concentran cerca de los lugares de residencia habitual de esta comunidad y tienen como principales clientes a miembros de la misma nacionalidad. En el caso español se incluirían dentro de esta tipología las carnicerías halal o los negocios de productos de alimentación chinos e iberoamericanos. Con la popularidad de ciertas cocinas exóticas y orientales estos negocios han aumentado considerablemente su clientela, incluyendo, cada vez más, a personas de la sociedad de acogida.

Una clase de establecimientos se dirige a la población del vecindario donde están situados, tanto a la población de la comunidad de origen como a la población autóctona. El ejemplo paradigmático, en el caso madrileño, sería el del pequeño comercio de ultramarinos y de alimentación regentado por chinos.

Otro tipo de negocios van dirigidos, fundamentalmente, al conjunto de las comunidades extranjeras residentes en una determinada zona. Un ejemplo significativo de este tipo de negocios son los locutorios (que se

Estudios Geográficos, LXIII, 248/249, 2002

$$
-576-
$$


dirigen al conjunto de la población extranjera) que combinan, igualmente, la venta de productos típicos y el envío de divisas.

Del total de establecimientos censados en Lavapiés (415) el 68\% (285) se dedican a la venta al por mayor de ropa, complementos y objetos de regalo. El resto de los establecimientos, a la restauración étnica (orientales, norteafricanos, turcos, etc.), a tiendas de alimentación varia, a carnicerías islámicas, a locutorios y servicios de envío de remesas, y a peluquerías étnicas, fundamentalmente.

En cuanto a la procedencia de los regentes de los negocios, aproximadamente la mitad, 51\%, son chinos, seguidos de bangladeses (15\%), iberoamericanos, norteafricanos, procedentes de Oriente Medio y Subsaharianos.

\section{Resultados}

Hasta ahora, la investigación sobre la economía étnica en la zona de Lavapiés ha puesto de menifiesto su centralidad. Los comercios de Lavapiés, regentados por extranjeros tienen, predominantemente, un alcance metropolitano. Son establecimientos de venta al por mayor, de productos elaborados tanto dentro como fuera de España. Lavapiés se aleja de la imagen de enclave étnico cerrado sobre sí mismo, ofreciendo, en cambio, la apariencia de un barrio dinámico y cosmopolita.

El predominio de las etnias asiáticas, sobre todo chinas y bangladesas, de reciente incorporación a la geografía española peninsular (de 1990 en adelante), resulta muy significativa, indicando la madurez comecial del área, capaz de atraer a importantes redes internacionales de comercio de productos semiperecederos. Por otra parte, queremos señalar que hemos llevado a cabo un censo de locales comerciales, no un censo de la población inmigrante de Lavapiés; es decir, los orientales no tienen por qué ser los habitantes más numerosos de la zona, del barrrio, del distrito, etc. Cuando contrastemos los datos de residentes y comerciantes de Lavapiés, esperamos obtener interesantes combinaciones.

Finalmente, es digno de reflexión que los inmigrantes hayan sustituido a los propietarios autóctonos, manteniendo básicamente, en esa zona que linda con el Rastro madrileño, el tipo de negocios preexistente. 


\section{BIBLIOGRAFÍA}

AJA FERNÁNDEZ, E. (2000): La inmigración extranjera en España: los retos educativos. Fundación «La Caixa», Barcelona.

ARANGO, J. (2000): «Becoming a country of Immigration at the End of the Twentieth Century: The case of Spain», en King, R.; Lazardis, G. y Tsardanidis, C., eds, Eldorado or Fortress? Migration in Southern Europe. McMillan Press. Londres.

ARANGO, J. y BALDWIN-EDWARDS, M., eds. (1999): Immigrants and the Informal Economy in Southern Europe. Frank Cass. Londres.

BAGANHA, M., ed. (1997): Immigration in Southern Europe. Celta. Oeiras. Portugal.

(2000): «Labour Market and Immigration. Economic Opportunities for Immigrants in Portugal», en King, R,; Lazardis, G,, y Tsardanidis, C,, eds,, Eldorado or Fortress? Migration in Southern Europe. McMillan Press. Londres.

BARRET, G., JonEs, T. y McEvoy, D. (1996): «Ethnic Minority Business: Theoretical Discourse in Britain and North America». Urban Studies, vol. 33, n. ${ }^{\circ} 4-5$, pp. 783-809.

BASSI, N. y JoHnson, M. (1996): Asia and White Businesses in the retail sector: a comparative analysis of development patterns. Center for Reseach in Ethnic Relations. Warwick.

BELtRÁN, J. (2000): «Empresa familiar. Trabajo, redes sociales y familia en el colectivo chino» Ofrim Suplementos. Número especial sobre mercado laboral e inmigración, pp. 129153.

BoNACICH, E. (1973): «A theory of middleman minorities», en American Sociological Review, vol. 37 , pp. $547-559$.

BORJAS, George J. (2000): Issues in the economics of immigration. University of ChicagoPress, Chicago.

CACHÓN, L. (1997): «Segregación sectorial de los inmigrantes en el mercado de trabajo en España», Cuadernos de Relaciones Laborales, n. $^{\circ} 10$

- (1999): Prevenir el racismo en el trabajo en España. IMSERSO. Ministerio de Trabajo y Asuntos Sociales.

DíEZ NiCOLÁs, J. (1999): Los españoles y la inmigración. Observatorio permanente de la Inmigración. IMSERSO. Madrid.

DolADo, J. J., Jimeno, J. F. y Dulce, R. M. (1997): «Los efectos de la inmigración sobre la demanda relativa de trabajo cualificado versus poco cualificado: evidencia para España», Cuadernos económicos del ICE, n. ${ }^{\circ} 63$, pp. 11-30.

FERNÁNDEZ CORNEJO, J. A. y AlgARRA, A. (2000): El mercado de trabajo en la Unión Europea, Ed. Pirámide, Madrid.

GIMÉNEZ, C. (1997): «La integración de los inmigrantes y la interculturalidad. Bases teóricas para una propuesta práctica», Arbor, n. ${ }^{\circ} 607$, pp. 119-147.

- (1998): Inmigración y multiculturalidad en Lavapiés. Informe de investigación. Proyecto «Inmigración, integración social y ciudadanía», dirigido por Emilio Lamo de Espinosa. Instituto Universitario Ortega y Gasset.

- (2000): «Inmigración y Multiculturalidad en Lavapiés: un marco interpretativo de los procesos urbanos de multiculturalización». Ponencia presentada al II Congreso sobre la Inmigración en España: España y las Migraciones Internacionales en el cambio de Siglo». Instituto Universitario Ortega y Gasset y Universidad Pontificia de Comillas. Madrid, 5-7 de octubre de 2000.

Gosh, B., ed. (2000): Managing Migration. Time for a New International Regime? Oxford University Press. Oxford.

HARRISON, M. y DAVIES, J. (1995): Constructing Equality: housing associations and minority ethnic contractors. SAUS Publications. Londres.

IzQUIERDO, A. (1996): La inmigración inesperada. Trotta. Madrid.

JONES, T. y McEvoy, D. (1992): «Ressources ethniques et egalités des chances: les enterprises

Estudios Geográficos, LXIII, 248/249, 2002

$$
-578-
$$


indo-pakistanaises en Grande-Bretagne et au Canada», Revue Européene des Migrations Internationales, n. ${ }^{\circ}$ 8, pp. 107-125.

JONES, T., BARRET, G. y McEvoy, D. (1994): «Labour intensive pratices in the ethnic minority firm», en Atkinson, J. y Storey, D (edits.), Employment, The Small Firm and the Labour Market. Routledge, pp. 172-205.

KING, R.; LAZARDIS, G. y TSARDANIDIS, C., eds. (2000): Eldorado or Fortress? Migration in Southern Europe. MacMillan Press. Londres.

KLOOSTERMAN, R. (1996): «Mixed Experiences: postindustrial transitions and ethnic minorities on the Amsterdan Labour Market», en New Community, vol. 4. pp. 637-654.

Light, I. (1972): Ethnic Enterprise in America. Business and Welfare among Chinese, Japanese and Blacks. University of California Press. Berkeley.

- (1984): «Immigrant and Ethnic Enterprises in North America», Ethnic and Racial Studies, vol. 7, pp. 195-216.

LÓPEZ DÍAZ, Julio y RIDRUEJo, Zenón J. (2000): Crecimiento y pensiones : el papel de la inmigracion. Universidad de Valladolid.

Marshald, T. y Bottomore, T. (1998): Ciudadanía y clase social. Alianza Editorial. Colección ciencias sociales. Madrid.

MARTínez VeIGA, U. (1997): La integración social de los inmigrantes extranjeros en España. Trotta. Madrid.

McEvoy, D. y Jones, T. (1993): «Relative economic welcomes. South Asian retailing in Britain and Canada», en Rudolf, R. y Morokvasic, M., eds., Bridging States and Markets. Sigma. Berlín.

McLaughis, C. y Jesilow, P, (1998): «Conveying a Sense of Community along Bolsa Avenue: Little Saigon as a Model of Ethnic Commerial Belts, International Migration, vol. 36, n. ${ }^{\circ} 1$, pp. $49-63$.

MENAHEN, G. y SPIRO, S. (1999). «Immigrants in a Restructuring Econmy: A partial test of Theories», International Migration, vol. 37, n. ${ }^{\circ} 3$.

Mingione, E. y QuASSOLI, F. (2000): «The Participation of Immigrants in the Underground Economy in Italy», en King, R., Lazardis, G. y Tsardanidis, C., eds, Eldorado or Fortress? Migration in Southern Europe. McMillan Press. Londres.

Monder, R., Sanghera, B., AbBas, T., Barlow, G. y Jones, T. (2000): «Ethnic minority business in comparative perspective: the case of indepdendent restaurant sector», en Journal of Ethnic and Racial Studies, vol. 26, n. ${ }^{\circ} 3$, pp. 495-510.

MonNET, N. (2000): «Prácticas urbanas y sociales de los grupos que conviven en el Casc Antic de Barcelona». Ponencia presentada al II Congreso sobre Inmigración en España: España y las Migraciones Internacionales en el cambio de siglo. Instituto Universitario Ortega y Gasset y Universidad Pontificia de Comillas. Madrid, 5-7 octubre de 2000.

Oc, T., TIESDELL, S. y MoYNiHAN, D. (1997): Urban regeneration and ethnic minority groups: Training and Business support in city challenge areas. The Policy Press. Area Regeneration Series. University of Bristol. Bristol

OFRIM SUPLEMENTOS (2000): Número especial dedicado a la inmigración y el mercado de trabajo. Consejería de Servicios Sociales. Comunidad de Madrid.

PAJARES, Miguel (1998): La inmigracion en España : retos y propuestas, Icaria, Barcelona.

Portes, A. y JEnSEn, L. (1987): «What's an Ethnic Enclave? The case for conceptual clarity», American Sociological Review, . $^{\circ} 52$, pp. 768-771.

PorTEs, A. (edit.) (1995): The Eonomic Sociology of Immigration: Essays on Networks, Ethnicity and Entrepreneurship. Russel Sage Foundation. Nueva York.

RAFIQ, M. (1992): «Ethnicity and Enterprise. A comparison of Muslim and non muslim owned asian business in Britain», New Community, vol. 19, n. ${ }^{\circ} 1$.

RAM, M. y HollidAY, R. (1993): «Relative merits: family culture and kinship in small firms», Sociology, vol. 27 , n. ${ }^{\circ} 4$. pp. $629-648$. 
RAM, M. (1994): Managing to Survive: Working Lives in Small Firms. Blackwell. Londres. RAm, M. y Deakins, D. (1996): «African-Caribbean in business», New Community, vol. 11, n. ${ }^{\circ} 1$.

RATH, J. (2000): «Outsider's Business: acritical review of research on immigrant entrepreneurship», en International Migration Review, vol. 34, n. ${ }^{\circ}$ 3, pp. 657-681.

SAssen, S. (1991): The Global City. Princeton University Press. Princeton.

- (1993): La movilidad del trabajo y del capital: un estudio sobre la corriente internacional de la inversión y del trabajo. Ministerio de Trabajo y Seguridad Social. Colección estudios. Madrid.

RESUMEN: Título: El negocio étnico, nueva fórmula de comercio en el casco antiguo de Madrid. El caso de Lavapiés. En este artícuo se presenta un desarrollo conceptual y metodológico para el análisis de las economías étnicas de los cascos históricos de las ciudades europeas. Además, de manera sucinta se publican los primeros resultados de su aplicación al estudio de la zona de Lavapiés, en el casco antiguo de la ciudad de Madrid. Pero la economía étnica urbana, tan interesante, no se desvela a la primera intentona. Por ello, los autores prometen futuras publicaciones sobre el mismo tema y lugar.

Palabras Clave: Economía étnica, Redes migratorias, Empleo y Autoempleo, Economía de las Migraciones.

ABSTRACT: Title: Ethnic business in the old district of Madrid. The case of "Lavapiés». The paper addresses the conceptual and methodological sides of the analysis of ethnic economies in the old districts of European towns. The case of Lavapiés is addressed, as a prototypical zone. Still, ethnic business is hard to unravel completely. Then, the authors announce later releases on the same topic and area.

KEYWORDS: Ethnic business, Migration networks, Employment and Self-employment, The Economics of Migration. 